\title{
Paper \\ Experience of a comprehensive pain care (CPC) clinic: from a Provincial General Hospital
}

\author{
Jayamaha $\mathbf{R}^{1}$ \\ Journal of the Ceylon College of Physicians, 2015, 46, 11-15
}

\section{Introduction}

Pain is an unpleasant physical and emotional sensation due to actual or potential tissue damage or described in terms of such damage. Acute pain is a symptom of an underlying disease. However, when it becomes chronic, and exceeds three months duration due to certain reversible and irreversible changes in the nervous system, it becomes a disease by itself. Most acute pains are nociceptive due to some form of tissue damage. However most chronic pains are secondarily neuropathic due to the above-mentioned changes, which are collectively called sensitization. ${ }^{1,3}$

Chronic pain is a disease by itself. It should be recognized as a non-communicable disease (NCD). If we look at its global incidence, chronic pain is the commonest NCD. Additionally, it has been more recently hypothesized that chronic pains and other NCDs are closely linked through a pathological process called metaflammation. The hallmark of metaflammation is that it is the result from environmental inducers and unhealthy lifestyles which activate the immune system that in turn damages various organsystems. Metaflammation has been linked to the occurrence of atherosclerotic diseases, chronic obstructive pulmonary disease (COPD), Alzheimer's disease, inflammatory bowel disease and some neuropathic pains which are interlinked. . $^{4,7}$

\section{Objectives}

Due to a paucity of data on the prevalence of chronic pain in Sri Lanka, we aimed to carry out a demographic survey through a point prevalence study of pain related complaints among general medical outpatient clinic attendees of a Provincial General Hospital.

\section{Methodology}

Permission was granted by the Medical Director to carry out a descriptive study at the Provincial General Hospital, Badulla. We used a pre-validated questionnaire that was pilot tested on a sample group

\footnotetext{
${ }^{1}$ Consultant Physician, Provincial General Hospital, Badulla, Sri Lanka. E-mail: drrohitha@gmail.com
}

of 25 volunteers from among unskilled hospital support staff.

Trained intern medical officers of the medical unit collected data by questionnaire based interviews of the first 40 follow up patients attending each medical clinic held on consecutive working weeks. Inclusion criteria were regular medical clinic attendees who were presenting as follow-up patients, while all new patient referrals were excluded. There were 14 medical clinics during the study period (24/01/2012 - 03/05/2012).

A total of 560 consenting subjects were interviewed. Out of these 24 data sheets were discarded due to incomplete data caused by communication barriers. The final sample consisted of 536 patients.

\section{Results}

Table 1 depicts the age stratification, distribution of gender and chief system of affection among the systematically recruited sample of adult follow up patients in the medical clinic of the Provincial General Hospital, Badulla over a period of 12 consecutive working weeks. There was a female preponderance with female: male ratio of $2: 1$. The predominant systems affected among patients were of the 'nervous system' and 'musculo-skeletal' systems, as determined by trained junior doctors who followed a structured evaluation of clinical history and case notes. A total of $357(67 \%)$ had pain as their chief complaint. However, when pain as a symptom, but not the chief complaint, was also included to the analysis chronic pain afflicted $391(73 \%)$ of the total sample of clinic follow ups.

\section{Commentary}

This data provides a clear picture that most of outpatient clinic attendees have chronic pain as a major cause of seeking hospital based clinic treatment. It is noteworthy that the majority of subjects interviewed were troubled by chronic pain arising from neurological and muscular-skeletal pathologies.

Despite possible selection bias in our patient sample that might have overestimated the problem, due to our unit's commitment to and interest in treating the chronic pain, the data shows there is a sizeable 
Table 1. Distribution of pain as the chief complaint among outpatients attending the medical clinic

\begin{tabular}{|c|c|}
\hline Age range (years) & $\begin{array}{c}\text { Number of patients (\%) } \\
\qquad N=536\end{array}$ \\
\hline$<20$ & $4(0.75 \%)$ \\
\hline $20-50$ & $123(23 \%)$ \\
\hline $50-80$ & $397(74 \%)$ \\
\hline$>80$ & $12(2.25 \%)$ \\
\hline \multicolumn{2}{|l|}{ Gender } \\
\hline Males & $178(33 \%)$ \\
\hline Females & $358(67 \%)$ \\
\hline \multicolumn{2}{|l|}{ System involved } \\
\hline Cardio vascular system & $91(17 \%)$ \\
\hline Nervous system & $247(46 \%)$ \\
\hline Musculoskeletal & $193(36 \%)$ \\
\hline Other & $5(1 \%)$ \\
\hline \multicolumn{2}{|l|}{ Chief complaint of pain } \\
\hline Yes & $357(67 \%)$ \\
\hline No & $179(33 \%)$ \\
\hline \multicolumn{2}{|l|}{ Duration of pain } \\
\hline$<3$ month & $145(27 \%)$ \\
\hline$\geq 3$ months (Chronic pains) & $391(73 \%)$ \\
\hline
\end{tabular}

proportion of outpatients with chronic pain whose complaints may be inadequately addressed in our busy state health sector outpatient medical clinics.

\section{Implementing a change}

Based on the findings of this survey, we established a model Comprehensive Pain Clinic (CPC) at the Provincial General Hospital, Badulla.

The existing diabetic center was used to conduct the CPC as it had all the resources required and also fell within the category of NCDs.

The floor plan of the CPC is described in Figure 1.

Management of chronic pain is based on a few key concepts

1. Diagnosis: of the underlying aetiology is essential as unplanned treatment of pain can lead to devastating outcomes by masking pain when it is the major presenting symptom of an underlying disease process.
2. Assessment of pain: is an important requirement as apart from the quality of pain being able to provide diagnostic clues, the quantification of pain is needed for chronic pain. Quantification is the key to escalate or de-escalate the treatment intensity and also to decide on the treatment modality.

3. Timing: of treatment is important for all chronic pains with minimal delay in order to minimize the development of otherwise difficult to treat neuropathic pain conditions.

4. Co-morbidities: many chronic pain conditions are associated with other NCDs. These conditions must be diagnosed and treated collectively. Additionally patients with chronic pain do develop psychological problems like anxiety and depression. Unless identified and treated holistically without delay, the pain issues can persist and become more complicated.

Based on the above targets we assessed, reassessed and managed our patients for their chronic pain, underlying diseases and co-morbidities.

The modalities we used to treat were

1. Noninvasive interventions

a. Non pharmacological - e.g. Exercise and physiotherapy

b. Pharmacological

\section{Invasive interventions}

i. Interventional pain procedures - e.g. Joint / enthesis injections

ii. Surgeries - e.g. Disc surgery for sciatica

How and when to combine these modalities was based on available guidelines (e.g. WHO analgesic ladder), ${ }^{8}$ available evidence and the local expertise and resources. Depending on the varying pain scenarios different combinations of the above named modalities were utilized.

The commonly encountered chronic pain problems in our dedicated CPC included:

1. Spinal pains (Neck and back pain)

2. Headaches and facial pain

3. Shoulder pain

4. Lower limb degenerative arthritis

5. Painful neuropathies

6. Enthesopathies like Golfer's and Tennis elbows and plantar fasciitis

7. Carpal tunnel syndrome

The throughput of patient numbers and pain treatment and/or interventions required over a period of 25 months from our CPC records are depicted in Table 2. 


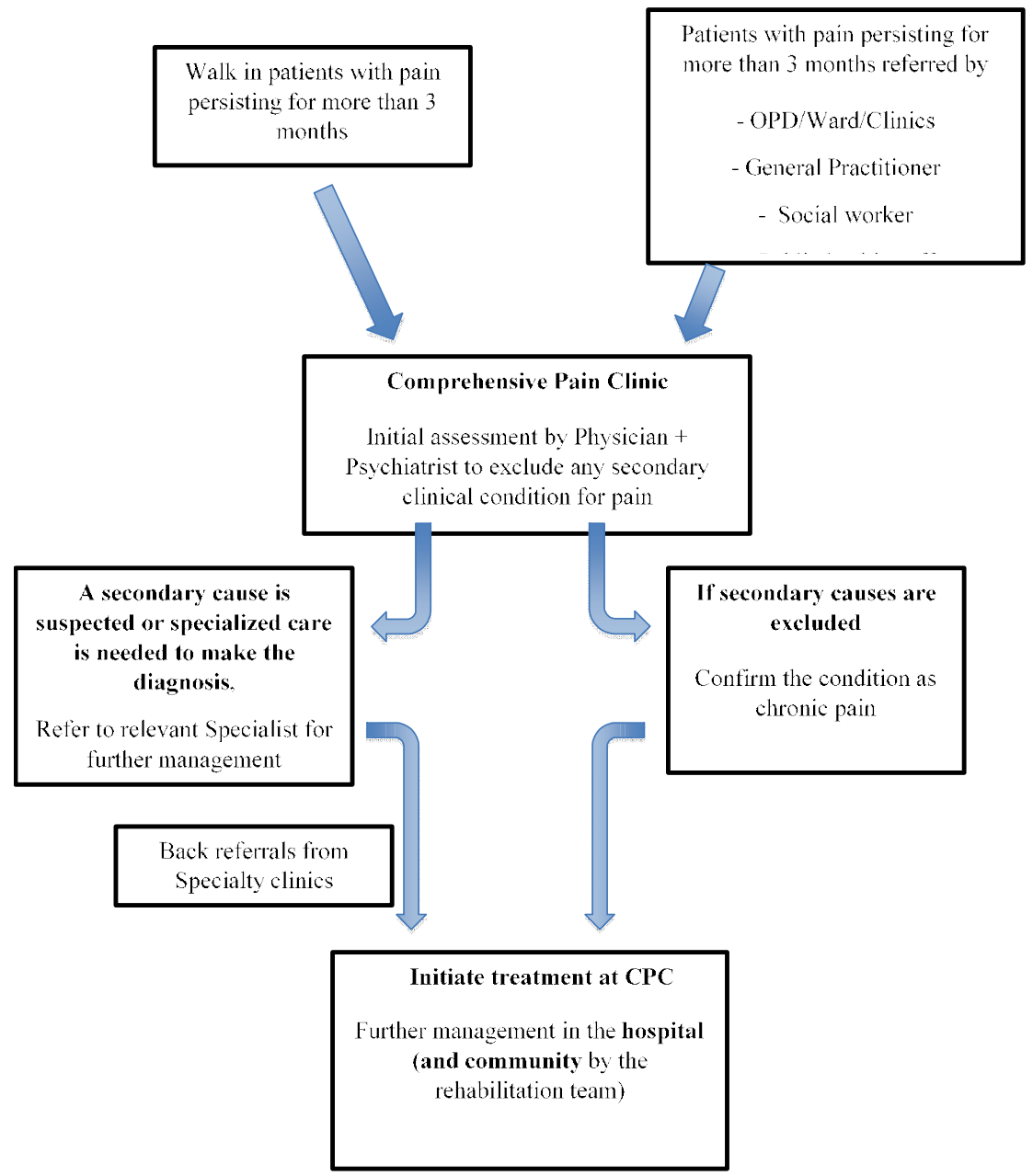

Figure 1. Flow-plan of the dedicated Chronic Pain Clinic

Table 2. Patient numbers and treatment given at the CPC from $01^{\text {st }}$ November 2012 to $30^{\text {th }}$ November 2014

\begin{tabular}{ccc}
\hline Year & $\begin{array}{c}\text { Number of patients } \\
(N=2124)\end{array}$ & $\begin{array}{c}\text { Number of } \\
\text { interventional pain } \\
\text { procedures performed (\%) }\end{array}$ \\
\hline $11 / 2012-12 / 2012$ (2 months) & 88 & $20(23 \%)$ \\
$01 / 2013-12 / 2013$ (12 months) & 1088 & $217(20 \%)$ \\
$01 / 2014-11 / 2014$ (11 months) & 948 & $226(23 \%)$ \\
Total (25 months) & $\mathbf{2 1 2 4}$ & $\mathbf{4 6 3} \mathbf{( 2 1 . 8 \% )}$ \\
Treatment given & & $\mathbf{8 0 \%}$ \\
Non Invasive & & $100 \%$ \\
a. Non pharmacological & & $1 \%$ \\
b. Pharmacological & & $20 \%$ \\
Invasive $\quad$ Interventional pain procedure & & $1 \%$ \\
\end{tabular}


Out of the 88 patients who attended the CPC in last 2 months of 2012, 20 (23\%) underwent an interventional pain procedure. Similarly out of 1088 and 948 who attended the CPC in 2013 and 2014, 217 $(20 \%)$ and $226(23 \%)$ underwent interventional pain procedures respectively.

Therefore it is noteworthy that on average about $22 \%$ of the patients required an interventional pain management (IPM) procedure for pain relief. Hence, IPM must be considered as an integral and indispensible part of chronic pain management. However the majority $(80 \%)$ of patients did not need any IPM procedure. This highlights what is required, not just an interventional pain clinic but an organized multi-disciplinary comprehensive pain clinic (CPC).

Our aim at the CPC is a holistic approach to the problem rather than an individualized approach. We consider the patient as a partner of the treatment process and make use of a group treatment approach rather than an individual patient care plan. The novel concepts of the holistic model and how they differ from the traditional model is highlighted in Table 3.

We so hope the concept of CPC would positively influence policy makers and planners on the current and emerging burden of chronic pain as an important NCD in parallel with what is experienced the world over. Even if we do not have our own outcome figures yet, we plan a re-audit. Studies around the world have demonstrated that almost $50 \%$ of conventionally treated patients require surgery or hospitalization compared to $16 \%-17 \%$ of CPC patients. ${ }^{9,10}$ Annual medical costs of an outpatient service have been shown to be reduced by $68 \%$ following the establishment of a
CPC service. ${ }^{9,10}$ Evaluating the average return to work rate from 20 different clinical studies demonstrates that on average $67 \%$ of CPC patients return to work compared to only $27 \%$ of non CPC patients..$^{9,10}$

Chief among the limitations we have encountered is the inability to implement a community based rehabilitation process. Therefore a national focus on the clinical burden of chronic pain and an effective and pragmatic program from within the existing health care service with recognition of the need for CPC with a multi-disciplinary approach is required in our national NCD program.

\section{References}

1. Egger G. In Search of a Germ Theory Equivalent for Chronic Disease. Preventing Chronic Disease 2012; 9: 110301.

2. Gureje O, et al. Persistent Pain and Well-being: A World Health Organization Study in Primary Care. Journal of American Medical Association 1998; 280: 147-51.

3. Stewart WF et al. Lost Productive Time and Cost Due to Common Pain Conditions in the US Workforce. Journal of American Medical Association 2000; 290: 2443-54.

4. Magni $G$ et al. Chronic Musculoskeletal Pain and Depressive symptoms in the National health and Nutrition Examination 1. Epidemiologic follow up study. Pain 1993; 53: 163-68.

5. Donovan $\mathrm{Ml}$, et al. When there is no benchmark: designing a primary care-based chronic pain management program from the scientific basis up. Journal of Pain and Symptom Management 1999; 18: 38-48.

6. Pain factsheet. Pain in America: highlights from a Gallup survey. Arthritis Foundation [homepage on the Internet] 1999. (Available from: http://www. arthritis.org/conditions/ speaking of pain/factsheet.asp)

Table 3. A comparison of the traditional and emerging models of chronic pain care

\begin{tabular}{ll}
\hline Traditional (Dualistic) Model & Emerging (Holistic) Model \\
\hline Medical or psychological focus & Additional Social and environmental focus \\
More clinician centered & More patient centered \\
Patient as recipient of treatment & Patient as a partner in treatment \\
Individual treatment approach & Individual and group treatment \\
Distracts recipient from active management & Involves recipient in active self-management \\
Potential for dependency/ complications & Limited dependency/complications \\
Limited benefits for limited time & Significant, long term benefits \\
Little or no attention to lifestyle & Significant attention given to lifestyle changes \\
Individual health perspective only & Population health perspective \\
\hline
\end{tabular}


7. Pain Facts and Figures: Incidence of Pain, as Compared to Major Conditions. www.painfoundation.org, January 2007.

8. World Health Organization. Traitement de la douleur cancéreuse. Geneva, Switz: World Health Organization 1987.

9. Loeser JD Comprehensive pain programs versus other treatments for chronic pain. The Journal of Pain 2006: 800-801.

10. Gatchel RJ Okifuji A. Evidence-based scientific data documenting the treatment and cost-effectiveness of comprehensive pain and programs for chronic nonmalignant pain. The Journal of Pain 2006: 779-93. 